\title{
Max Weber: sugerencias para una "reconstrucción"
}

\author{
Ricardo F. Crespol \\ Universidad Nacional de Córdoba (Argentina)
}

Recently have been advanced some variations to the standard interpretation of Max Weber's work. Among these variations there is a non-sociological view of weberian corpus. Here I try to show some implications of this new position for the methodology of social sciences, and offer a bibliographic overview of the topic.

El objetivo de esta nota es presentar algunas variantes a la interpretación habitual acerca del "tema central" de Max Weber y de su propuesta metodológica para las ciencias sociales. Decimos "presentar", pues no pasaremos de ello. La demostración de la validez de estas variantes sería una tarea enorme, que no pretendemos abarcar en esta breve nota. En la misma sólo daremos las indicaciones acerca de los autores y sus ideas principales. Acá no probamos nada: sólo señalamos las posibilidades nuevas y sus referencias, de modo de suscitar y facilitar investigaciones más profundas. Sin embargo, a pesar de este alcance limitado, pensamos que vale la pena este trabajo, como modo de contribuir a romper con la vigencia de una cierta opinión a-crítica acerca de las ideas nucleares del célebre pensador alemán. Por otra parte, Weber sigue siendo actual; sus problemas son los contemporáneos: la interpretación de la situación del hombre en la modernidad, la democracia y el poder, el método y la valoración en las ciencias sociales ${ }^{2}$. También por esto nos parece.

1 Agradezco las observaciones de un dictaminador anónimo.

2 Raymond ARON, titula uno de sus apartados de la parte dedicada a Weber en Las etapas del pensamiento sociológico, Siglo Veinte Editores. Buenos Aires 1981, 
importante dar a conocer otras posibles interpretaciones recientes de su pensamiento.

Cuando hablamos del tema central de Weber nos referimos a su diagnóstico de la modernidad, y del "desencantamiento" del mundo, como un proceso de racionalización ${ }^{3}$. A esto se debe unir su afirmación de una ética de la responsabilidad, y una teoría del poder en la que la democracia parece más bien circunstancial. Esta combinación constituye una postura que es considerada por algunos, especialmente lo más radicales de la escuela de Frankfurt, como un intento de legitimación ideológica. En cuanto a la propuesta metodológica, apuntamos a su imposición de la neutralidad valorativa como criterio de cientificidad en el, campo social. Pensamos que ambos temas son lo suficientemente conocidos y que no es necesario abundar en los mismos.

La imposibilidad de abarcar con profundidad la riqueza del pensamiento de Weber es evidente. Muestra de lo cual es la enormidad de la bibliografia sobre la cuestión. Walter G. Runciman decía en 1971 que los trabajos sobre los aspectos metodológicos en Weber ascendían a unos 600 títulos $^{4}$. G. Roth y W. Schluchter agregan que en la primera mitad de los 70 se escribieron otros 100 ensayos más por año ${ }^{5}$. Han pasado otros 20 años, por lo que conocer

T. II, pp. 219-316 (Les étapes de la pensée sociologique, Gallimard. Paris 1965, trad. Anibal Leal), "Weber, nuestro contemporáneo" (pp. 302 y ss.). También Wolfgang SCHLUCHTER, profundo conocedor de la obra de WEBER, señala que "sus problemas son aún, al menos en parte, los nuestros, y no han sido resueltos": Gunther Roth y W. Schluchter, Max Weber's Vision of History. Ethics and Methods, University of California Press. Berkeley-Los Angeles-London 1979, p. 13.

3 Cfr., e.g., Jürgen HABERMAS, El discurso filosófico de la modernidad, Taurus. Buenos Aires 1989 (Der Philosophische Diskurs der Modern, Suhrkamp Verlag. Frankfurt 1985. trad. M. Jiménez Redondo), pp. 11-15.

4 Cfr. Walter G. Runciman: A Critique of Max Weber 's Philosophy of Social Sciences. Cambridge University Press. Cambridge 1972, p. vi.

5 Cfr. Max Weber's Vision..., p. 1. 
a fondo la bibliografia sobre el tema supondría una dedicación específica al mismo que no es la mía. Recientemente, además, se ha publicado un importante libro que agrupa varios textos de Wilhelm Hennis. Su título es muy sugestivo: Max Weber: Essays in Reconstruction; de alli hemos tomado el de-esta nota. Hennis, que conoce a fondo la obra de Weber, afirma de él que "prácticamente nadie ha sido tan desafortunado por la mala comprensión De otro modo nunca se hubieran escrito las bibliotecas que hay sobre las 'tesis de Weber". "Por tanto, Weber debe ser leído fresco y sin prejuicios. Y esto significa todo el corpus de su obra"7. Por eso acá, como decíamos antes, sólo nos limitaremos a dar cuenta brevemente de algunas interpretaciones de su "tema central" y de su pensamiento metodológico que, aunque difieren de las habituales, son muy atendibles.

\section{El "tema central" de Weber.}

Este seria el primer punto. Respecto al mismo, Hennis nos presenta un Weber nuevo e insospechado, que no sería el padre de la Sociología contemporánea, sino que se hallaría en la tradición de las ciencias morales $\circ$ prácticas clásicas ${ }^{8}$. Estaria interesado principalmente en la naturaleza del hombre y el orden de su vida en las circunstancias de la modernidad ${ }^{9}$. Contrasta notablemente con la interpretación habitual, el mismo Hennis lo afirma ${ }^{10}$; pero, tal como

6 Wilhelm Hennis: Max Weber. Essays in Reconstruction, Allen \& Unwin. London 1988. (transl. by Keith Tribe).

7 Max Weber. Essays..., pp. 27 y 22.

8 Cfr. Max Weber. Essays..., pp. 103, 104 donde afirma: "Weber belongs to the late tradition of practical science; and he finds a place in the pre-history of modern social science only if his central questions and concerns are neglected".

9 Cfr. Max Weber. Essays..., pp. 35, 36, 43, 44, 61, 69, 73, 90, 108. "The 'cultural problems of man' remain the object of his work. And this means: the problems arising form the insertation of man (Mensch), a being capable of social action, in social constellations which in turn form these persons, develop their capacities or alternatively deform them up..." (69).

10 HENNIS menciona entre los que han roto con WEBER, a Georg LUKACs y Herbert MARCUSE, por una parte y a Leo Strauss y Eric Vógelin, por otra: Max Weber. Essays..., p. 21. 
dice Keith Tribe en la introducción del traductor, su erudición es tal y la argumentación tan convincente, que no se puede dejar de lado esta posibilidad. Sin embargo, sostiene Hennis, la suya no es una interpretación nueva. Sólo pretende restablecer lo que era relativamente claro para los intérpretes originales: Marianne Weber, Karl Lowith, Siegfried Landhust y Albert Salomon" ${ }^{11}$

Ante todo, Weber no es sólo ni fundamentalmente un sociólogo. Tiene una formación inicial en derecho, se estrena en las lides académicas con la cátedra de economía política en la Universidad de Friburgo, sus conocimientos históricos son notables, incursiona en cuestiones políticas, y también, por supuesto, metodológicas. Sería a propósito de sus definiciones en este último campo, que surgiría la' mayor pureza de sus investigaciones sociológicas. Schluchter, sin embargo, ve en nuestro autor más un filósofo que un sociólogo. Dicho de modo positivo, Weber fue mucho más que un sociólogo o un economista. La obra de Weber no está caracterizada por la presencia de material de una sola disciplina específica, de la cual deriva su coherencia: todavía está inmerso en el sector más amplio de las ciencias de la cultura, tan desarrollado en Alemania la segunda mitad del siglo pasado.

Sin duda que no es incorrecto, piensa Hennis, afirmar que el tema principal de Weber fue el proceso de racionalización; pero es una afirmación incompleta. Lo que final y centralmente interesa a Weber es el desarrollo de un modo de ser humano, de la "humanidad" -Menschentum-, en el sentido de la cualidad, modo de ser, virtudes, del hombre en el momento concreto de la modernidad; para lo que le ayuda y es clave el concepto de racionalización. En la introducción de su célebre ensayo La ética protestante y el espiritu del capitalismo, queda claro que lo que le interesa averiguar y explicar es un ethos del hombre contemporáneo ${ }^{12}$. Hennis va siguiendo este interés a lo largo de la desordenada obra de Weber, y concluye: "Resumamos: ¿cuál

11 Cfr. Wilhelm HennIs: Max Weber. Essays..., p. ix.

12 Cfr. Weber: L'éthique protestante et l 'esprit du capitalisme. Plon. Paris 1967, p.24. 
fue el interés central de Weber en la mayoria de sus famosos estudios? (...) Menschentum mediante una investigación histórica diferencial! Porque el interés constante y exclusivo de las 'ciencias culturales' eran los problemas 'cualitativos' y 'valorativos' y Menschentum era la palabra que, desde mediados del siglo pasado, el idioma alemán había elegido para expresar el interés 'cualitativo' en la historia de la Humanidad"13. Se trata de una preocupación por un problema antropológico y ético: "está interesado [sigue Hennis] en la 'suerte del hombre' y, por tanto, la problemática es por naturaleza antropológica, caracterológica y ética"14. Efectivamente, estos rasgos de la interpretación de la obra de Weber propuestos por Hennis, son realmente novedosos respecto a la habitual.

\section{La metodología de las ciencias sociales.}

Pasemos a este segundo aspecto. Es bien conocido que la condición principal de cientificidad impuesta por Weber a las ciencias sociales es la Wertfreiheit, libertad de valoración, neutralidad ética o avaloratividad. Weber es muy claro en este aspecto en sus diversos escritos, y pienso que no vale la pena perder el tiempo con citas al respecto. Basta con poner el ejemplo de sus dos famosos ensayos: "El significado de la libertad de valoración en la Sociología y la Economia" y "Sobre la objectividad en las Ciencias Sociales"15. También podemos mencionar en este sentido su conferencia "Ciencia

13 Wilhelm Hennis: Max Weber. Esssays..., pp. 43-44.

14 Wilhelm HenNis: Max Weber. Essays..., p. 73.

15 Reunidos en WeBer: Sobre la teoría de las ciencias sociales, Península. Barcelona 1971 (trad. M. Faber-Kaiser). En inglés en The Methodology of the Social Sciences (translated and edited by Edward Shils and Henry Finch). The Free Press. Glencoe, IL 1949. El ensayo sobre la objectividad -"Die Objektivität sozialwissenschaftlicher und sozialpolitischer Erkenntnis"- se publicó primero en los Archiv für Sozialwissenschaft und Sozialpolitik en 1904, con ocasión de que el mismo WEBER, SOMBART y JAFFÉ se hicieran cargo de su edición, y el otro ensayo - "Der Sinn der Wertfreiheit der soziologischen und okonomischen Wissenschaften"- fue preparado en 1914 y publicado en una nueva versión revisada en Logos en 1917. Ambos fueron reeditados en Gesammelte Aufsätze Wissenschaftslehre, 2nd. ed. Tübingen 1951. 
como vocación", en la que afirma que no se puede esperar de la ciencia ninguna respuesta acerca del sentido de la vida y los valores ${ }^{16}$ Esta postura weberiana ha marcado el rumbo futuro de la intencionalidad metodológica de las ciencias sociales de alli en más.

Sin embargo lo que hemos dicho quedaría incompleto, si no agregáramos que la imposición del criterio de neutralidad respecto a los valores no es absoluta. Ello por dos motivos. El primero de ellos es sostenido por muchos autores: a la hora de hacer ciencia social el mismo Weber no habría sido coherente y no habría respetado dicho principio. El Weber de 'las monumentales investigaciones sociológico-históricas no sería el de la estricta Wertfreiheit. En las mismas se confundirian una evidente intencionalidad de objetividad con una implícita asunción de ciertos valores. Así por ejemplo, Wilhelm Hennis dice que los 'Escritos políticos' de Max Weber, ante todo los escritos constitucionales de los años de la guerra, hacen caso omiso de su posición al respecto ${ }^{17}$. Eric Vögelin llama a esta actitud de Weber un "positivismo con lamentaciones". Además ensaya una explicación acerca del motivo por el que no se habria animado a dar el paso decisivo hacia una ciencia del orden; pero llega a decir que con la obra de Weber el positivismo llega a su fin y deja abiertos los caminos para la restauración de la ciencia política clásica ${ }^{18}$. Leo Strauss, por su parte, afirma que "los juicios de valor, que tenian prohibida la entrada por la puerta principal de la ciencia política, de la sociología o de la economía, terminan ingresando por la trasera"19.

16 "Wissenschaft als Beruf". Conferencia publicada junto a "Politik als Beruf" ("La política como vocación") en El político y el científico, Alianza. Barcelona 1969. 2a. ed. Sobre su fecha, cfr. RoTH and SCHLUCHTER: Max Weber's Vision of History..., pp. 112 y ss.

17 Cfr. WEBER, "Politica y filosofĩa práctica", en El político y el cientifico..., p. 143. Cfr. también la referencia a esta cuestión hecha por Ronald INGLEHART, "Coercion and Consent: (...).", en Peter KosLowskI (ed.): Individual Liberty and Democratic Decision-Making. J.C.B. Möhr. Tübingen 1987, p. 181.

18 The New Science of Politics. University of Chicago Press. Chicago 1952, Introduction, parágrafos 3 y 4 , passim.

19 What is Political Philosophy? and Other Studies. The Free Press. Glencoe, IL 1959 , p. 21. 
Más recientemente, Lawrence Scaff ha dicho que "la misma argumentación metodológica de Weber asume un papel diferente dependiendo del contexto específico"20. Ludwig von Mises también se queja de ello: "Max Weber [señala en sus Grundprobleme der Nationalökonomie de 1933] combatió expresamente esta confusión [el juicio de valor que identifica acción racional y correcta]; sin embargo, (...), cayó repetidas veces en la misma en otros pasajes de sus escritos"21.

La segunda "relativización" de la Wertfreiheit es la efectuada por el mismo Weber, como parte de su misma teoría de las ciencias sociales. Ésta ha dado lugar a una extensa literatura sobre el tema. Por una parte los valores están involucrados en la formación de los conceptos de las ciencias sociales. Es decir, el mismo contenido está determinado por valores. "Los problemas de las disciplinas empiricas deben resolverse, por supuesto, de modo no 'evaluativo'... Pero los problemas de las ciencias sociales deben seleccionarse por la relevancia valorativa de los fenómenos abordados"22. Weber se cuida muy bien de distinguir entre relevancia valorativa (Wertbeziehung) $y$ juicio de valor (Werturteil). Sin embargo, en opinión de muchos autores, esta distinción tiene muchos problemas y aunque Weber busque la objetividad finalmente caeria en un cierto relativismo. "En las ciencias de la cultura [afirma] la construcción de conceptos depende del planteamiento de los problemas, y este último planteamiento varía según el contenido de la cultura"23. Lo social requiere una determinación valorativa para delimitar un problema cientifico. Se necesita una selección regida por los valores, que son variables. "Las mismas [todas las ideas valorativas] son [dice Weber]

20 "Historicism in the German Tradition of Social and Economic Thought", en Peter Koslowski (ed.), The Theory of Ethical Economy in the Historical School. Springer Verlag. Berlin-Heidelberg 1995, p. 320.

21 Epistemological Problems of Economics. D. van Nostrand. Princeton-TorontoLondon-New York 1960, p. 93 (Trad. por George Reisman). (Grundprobleme der Nationalökonomie. Gustav Fischer. Jena 1933).

22 The Methodology..., p. 21.

23 The Methodology..., p. 105 (p. 82 en edición española). 
por naturaleza, históricamente variables, en función del carácter de la cultura y de las ideas que regulan el pensamiento de los hombres"24. Pero no sólo depende de los valores de la cultura analizada sino también de los del investigador mismo. "No existe un análisis absolutamente 'objectivo' de la cultura, dice, (...) de los fenómenos sociales, independiente de puntos de vista especiales y parciales, según los cuales -expresa 0 tácitamente, consciente 0 inconscientemente- son seleccionados, analizados y organizados por fines expositivos. El motivo de esto reside en la característica del fin cognoscitivo de toda investigación en ciencias sociales, que apunta a trascender el tratamiento puramente formal de las normas legales o convencionales que regulan la vida social". "Todo conocimiento de la realidad cultural, como puede observarse, es siempre conocimiento desde puntos de vista particulares". E, "indudablemente, todas las ideas valorativas son 'subjetivas" 25. Hoy dia estas opiniones están bastantes generalizadas a nivel de epistemología de las ciencias sociales.

Michael Lesnoff, afirma en este mismo sentido, que "en un nivel exclusivamente verbal (...) Weber creía que tanto la ciencia natural como la social podían y debian ser libres respecto a los valores, [pero] a un nivel un poco más profundo (.. ) Weber sostenia que las ciencias sociales debian ser también relevantes respecto a los valores, es decir, los conceptos deben reflejar inevitablemente los intereses de los científicos sociales"26. Karl Otto Apel, a su vez, dice que "hay un área en la que Max Weber (...) transgrede el límite entre lo racional y lo irracional o entre la ciencia libre de valoración y los valores comprometidos normativamente. No es accidental que esta área sea la de la ciencia y su historia"27. La objetividad tan ansiada se pierde en

24 The Methodology..., p. 84.

25 The Methodology..., pp. 72, 81, 83 .

26 Michael LeSNOFF: "Technique, Critique and Social Science", en S. C. BRowN (ed.) Philosophical Disputes in the Social Sciences, The Harvester Press. SussexHumanities Press. New Jersey 1979, p. 95.

27 "Toward a Reconstruction of Critical Theory", en S. C. Brown, Philosophical Disputes..., p. 136. 
un relativismo. Como dice Barry Hindess, "la arbitrariedad teórica implícita en la epistemología de los tipos ideales asegura que la evaluación de la utilidad de los conceptos tipo no puede ser objectiva (...). Una vez que se convoca a los valores para protagonizar un papel teórico, la 'objectividad', la 'libertad de valoración' y todo eso se cae por la borda. La concepción weberiana de la objectividad científica es un imposible lógico; contradice los conceptos fundamentales de su epistemologia"28. También Schluchter observa estos problemas: "Weber ha sido llamado un nihilista [por ejemplo, por Leo Strauss ${ }^{29}$ ], un relativista, y un decisionista. Lo es todo ello si creemos en la existencia y discernibilidad de un significado objetivo del mundo" 30 . Seria largo y no tiene sentido aquí seguir toda la interpretación de Schluchter. Lo mismo podriamos decir de Runciman, quien también advierte este asunto ${ }^{31}$. Cerramos esta cuestión con una cita de Raymond Aron, cuyas observaciones sobre Weber continúan siendo actuales y perspicaces: "si cada reconstrucción tiene carácter selectivo y está regida por un sistema de valores, habrá tantas perspectivas históricas o sociológicas como sistemas de valor utilizados en la selección"32. En definitiva, la Wertfreiheit no es ni puede ser estricta en el campo de las ciencias humanas. Y esto, según nuestro punto de vista, no constituye ningún defecto, sino algo sumamente realista que debe ser asumido y que no disminuye el carácter cientifico de dichas disciplinas.

Respecto a este asunto, Hennis nos vuelve a sorprender. En otro de sus recientes, concienzudos y documentados estudios nos dice "que no se puede entender la pasión con la que Weber sostenía el postulado de la libertad valorativa si se considera que éste tiene un fundamento

28 Barry Hindess: Philosophy and Methodology in the Social Sciences, The Harvester Press. Sussex 1977, p. 38. Cfr. también las pp. 24, 33-9, 48 y 232.

29 Cfr. Natural Right and History. The University of Chicago Press ChicagoLondon 1953, pp. 42 y ss.

30 SCHL UCHTER. Max Weber's Vision..., pp. 58-9.

31 SCHLUCHTER. Max Weber's Vision..., pp. 37 y ss., 50, 52, 60.

32 SCHLUCHTER. Max Weber's Vision...,p. 235. Cfr. también su Introduction a Le savant et le politique. Plon. Paris 1959, que reúne "Politik als Beruf" y "Wissenschaft als Beruf". 
'lógico-metodológico'". Se trataría más bien de una cuestión de libertad académica. El principio de libertad valorativa tiene primariamente una intención pedagógica, que surge de su lucha contra las arbitrariedades de las políticas académicas alemanas de su época: "en Alemania la 'libertad de la ciencia' existe en el ámbito de la aceptabilidad política y eclesiástica $-\mathrm{y}$ nunca fuera de dicho ámbito-". La 'libertad de valoración' es interpretada como 'imparcialidad'33.

Otro rasgo que queremos señalar finalmente de la metodología de Weber, y que vemos estrechamente conectado con los anteriores, es el carácter contingente, probable e inconcluso de los conocimientos de las ciencias humanas ${ }^{34}$. Estas características se manifiestan, por ejemplo, en el carácter casi meramente instrumental de los tipos ideales. No hay que confundirlos con la realidad ni con lo que se debe hacer. Pueden cambiar con las culturas y sus evoluciones. También se muestran en la parcialidad y probabilidad de las relaciones causales ${ }^{35}$. Esta es la situación de, entre otras ciencias sociales, la economía. La que, además, no se puede conformar sólo con un estudio causal teórico, sino que debe considerar también lo cultural e histórico, sin dejar por ello de ser ciencia.

En resumen, Weber impone la condición de avaloratividad como criterio de cientificidad, pero él mismo la relativizaría y saltaría. Por otra parte, Weber reconoce el carácter provisorio y contingente del conocimiento de las ciencias sociales, lo que no disminuye su cientificidad. Como decía Vögelin, a pesar de que para él no tenga esta intención, Weber está preparando las bases para un retorno al esquema clásico de las ciencias humanas, que eran morales.

33 "The pitiless 'sobriety of judgement': Max Weber between Carl Menger and Gustav von Schmoller-the academic politics of value freedom", en History of the Human Sciences, 4/1, 1991, p. 34 y passim. Cfr. también Max Weber, Essays..., p 161 .

34 Cfr. Max Weber. Essays..., pp. 227 y ss.

35 Cfr. HINDEss: The Methodology..., pp. 43 y ss., 89 y ss. Tambiẻn ARON, Las etapas del pensamiento..., pp. 244 y ss. y Runciman, A Critique of Max Weber's..., p. 36. 


\section{Conclusiones para las ciencias sociales.}

Una de las fracturas provocadas por la modernidad se manifiesta en el planteamiento epistemológico y metodológico de las ciencias sociales. El corte entre el ser y el deber ser conduce a un tratamiento teórico y neutro de las cuestiones prácticas, a una prescindencia de los fines en ámbitos en que estos son esenciales. La política, desde entonces, no es más aquel estudio que permitía determinar los fines debidos a la sociedad y los mejores medios para alcanzarlos, sino una descripción fenomenológica de los comportamientos sociales o una teoria sobre el poder. El derecho deja de ser el saber acerca del objeto de la virtud de la justicia, transformándose en un cuerpo de normas coherentes. La economía no es más, como la pensó Aristóteles, la actividad y ciencia del uso de los bienes adecuado a la vida virtuosa (Política I, 9), sino una ciencia de la elección más beneficiosa, sin un fin predeterminado. Los fines han quedado marginados de la ciencia, como elementos irracionales que sólo pueden ser tomados como datos que nos vienen de fuera. La razón científica sólo es aplicable a los medios. Los fines son cuestión de una decisión que excede el ámbito científico. La avaloratividad, como hemos visto, se transforma en una condición de cientificidad. Jürgen Habermas atribuye un papel importante a Weber en esta disolución, cuando dice que "desde que Max Weber clarificó la llamada disputa sobre el juicio valorativo (...), las ciencias sociales se han desligado completamente de los elementos normativos, de la ya olvidada herencia de la política c̀lásica -asi por lo menos lo perciben ellas, como evidencia científicoteórica" ${ }^{36}$. Esta desconexión de la ciencia respecto a los fines lleva de modo automático a otra entre las mismas ciencias sociales. Ellas se transforman en un conjunto de saberes particulares que se amontonan uno junto a otro sin la subordinación a la política que les daba unidad. Carecen de una orientación común que les marque un rumbo seguro $^{37}$. También Strauss, desde otra postura, piensa que Weber ha

36 WeBer: Teoria y praxis. Sur. Buenos Aires 1966, p. 10 , (trad. D. J. Vogelmann)(Theorie und Praxis, Hermann Luchterhand Verlag. Neuwied am Rhim-Berlin 1963).

37 Cfr. Wilhelm HenNis, Politica y filosofía práctica, pp . 54, 147 y passim. 
influido mucho en este proceso, que obedecería, en el fondo, a un escepticismo gnoseológico ${ }^{38}$. La reacción frente a esta pérdida de cientificidad de la política clásica, ha surgido desde la misma filosofia política. Algunos "precursores" como Leo Strauss, Eric Vögelin, Hannah Arendt y varios más, preanunciaron lo que se transformó en un movimiento de "rehabilitación de la filosofia práctica" en el que podemos nombrar a pensadores de diversas procedencias como Manfred Riedel, editor de una importante obra colectiva sobre el particular $^{39}$, Helmut Kuhn, Hans Georg Gadamer y Robert Spaemann. También hay muchos otros pensadores que sin poder agruparse bajo este título sostienen ideas similares. Las ciencias sociales van más lentas, como siempre sucede, en la aplicación de este nuevo paradigma. La fuerza del anterior es grande. Probablemente esta tarea llevará mucho tiempo. Supone un cambio de mentalidad muy fuerte hacia un modo de pensar ajeno para la mayoría de los científicos sociales. Pero parece necesario tratar de seguir las argumentaciones de aquellos pensadores en orden a superar los problemas, puesto que la solución para una complexión e integración de las ciencias sociales, radica en su reunificación a través de la consideración de los aspectos morales que inciden en las mismas.

Por lo que opinan Habermas o Strauss, parecería que Weber ha sido uno de los causantes de la disolución de la moralidad de las ciencias sociales. Sin embargo, si se vuelve a estudiar con atención su pensamiento, podría suceder, como sugiere Hennis, que el mismo Weber ayudara, al menos en parte, a la empresa de la recuperación de su practicidad. Lo que hemos intentado hacer en esta breve nota es advertir acerca de esta posibilidad, señalando algunos de los estudios que siguen esta línea, como modo de facilitar algunas hipótesis para un posible programa de investigación.

38 Cfr. Strauss: Natural Right and History..., pp. 22-3.

39 Manfred RIEDEL: Rehabilitierung der praktischen Philosophie. Rombach. Freiburg 1972-4. 
Copyright of Tópicos. Revista de Filosofía is the property of Universidad Panamericana and its content may not be copied or emailed to multiple sites or posted to a listserv without the copyright holder's express written permission. However, users may print, download, or email articles for individual use. 